\title{
3D Assessment of Irreversible Electroporation Treatments in Vegetal Models
}

\author{
Q. Castellví ${ }^{1}$, J. Banús ${ }^{1}$ and A. Ivorra ${ }^{1,2}$ \\ ${ }^{1}$ Universitat Pompeu Fabra, DTIC, Barcelona, Spain \\ ${ }^{2}$ Serra Húnter Fellow
}

\begin{abstract}
Ablative techniques based on irreversible electroporation (IRE) have emerged as an effective cancer therapy. In these treatments electrical pulses are delivered in order to induce irrecoverable damage to the cell membranes, thus, causing cell death. An interesting feature of electroporation treatments is the capability to predict shape and extension of lesions by means of mathematical simulations. Those prediction methods have been refined thought in-vivo experimental observations. Aiming at minimization of animal experiments, vegetal models have been used to easily observe the IRE outcomes. However, these observations are limited at the surface of the tissue. Here we present an improved method able to observe the inner parts of the tissue and therefore evaluate in three-dimensions the results of IRE using potatoes as vegetal models. The technique consists in using a dye solution to enhance the IRE area in sliced potato tubers. After slice digitalization, the electroporated area is automatically identified and the resulting treated volume is calculated. In addition, a threedimensional reconstruction of both healthy tissue and IRE volume is generated. The proposed evaluation technique was used to assess different pulse protocols outcomes. Numerical simulations had been carried out to compare the numerical predictions to the experimental observations. The obtained results show a clear match between experimental and simulated volumes confirming the reliability of the proposed method.
\end{abstract}

Keywords- Irreversible electroporation, In-vivo assessment, Vegetal model, Potato, Three-dimensional.

\section{INTRODUCTION}

Ablative techniques based on irreversible electroporation (IRE) have emerged as an effective alternative to other minimal invasive techniques such as radiofrequency ablation, microwave ablation or cryosurgery. IRE treatments are performed by delivering pulsed electric fields of high magnitude across electrodes. This causes irreversible damage on cell membranes, thus, causing cell death [1].

An interesting feature of electroporation treatments is the capability to predict shape and extension of the produced lesion using mathematical modeling. In those models, which are frequently solved as computer simulations based on numerical methods, it is assumed that a tissue region is affected by IRE once the electric field magnitude is higher than a certain threshold value.

The IRE threshold is a critical parameter in treatment planning. These values are commonly determined through experimental studies [2, 3]. In those experiments, the selected threshold value is the one which better fits the observed ablation area. Nevertheless, a certain miss-match is commonly observed between mathematically predicted and real ablated area. This discrepancy may be caused by multiple factors such as tissue fibers, tissue organization, cell orientation or cell shape.

Although IRE treatments protocols have been extensively studied, further studies are required in order to improve the prediction methods and the IRE protocols. However those studies could involve a high number of animals, histological observations and its associated costs. With the aim of exploring the electroporation outcomes with an inexpensive model and to follow the replacement concept of the 3Rs, alternative models had been proposed $[4,5]$.

The potato is a good model often used to assess the IRE outcomes. Those tubers are commonly employed since the tissue affected by IRE will become darker in a couple of hours. Furthermore potato shows a quite homogeneous and isotropic tissue structure which simplifies the studies. This technique of observation has been employed in multiple occasions to study the relation between applied protocols and IRE outcomes [6] and in order to compare predicted outcomes with the real ones [5].

The blackening process of electroporated potatoes is attributed to an accelerated oxidation produced by enzymes released during the cell lysis [7]. It implies that parts of the potato closest to the surface, which are in direct contact with the air, will be darker than inner parts despite being equally affected by IRE. In addition, the treatment outcomes are commonly observed about 12 hours after the pulse application. It can produce a desiccation of the sample which could mean a change of the morphology and, consequently, an error of outcomes measurements. It would therefore be necessary an alternative method to identify the electroporated area in those vegetal models.

Potatoes have a high compact tissue structure with a high density of cells. When IRE occurs, the cell death entail a turgor pressure loss [8] changing the mechanical properties of the tissue. The loss of turgidity in the electroporated areas implies an increase on tissue permeability to liquid. Taking advantage of this effect, here we propose the use of a common food colorant to enhance the irreversibly electroporated areas. 
This improved observation technique allows to observe fine slices regardless the desiccation effect. Thus, it is possible to observe the inner parts of the potatoes to assess the tridimensional outcomes of applied electroporation protocol.

In this paper, we report the treated volumes observed for a sort of applied pulse protocols using this new approach. Those values are also compared with numerical prediction to evaluate the goodness of the observations.

\section{Materials AND METHODS}

\section{A. Electroporation Setup}

A total amount of sixty potatoes were used in the experiment. Each potato had treated with a single treatment applied by means of needle shape electrodes.

The electrodes used during the experiments consist on two parallel stainless steel cylinders of $1.27 \mathrm{~mm}$ separated $15 \mathrm{~mm}$ mounted on an ABS plastic holder. These electrodes were inserted, through the peel, reaching the dip part of the potatoes where IRE treatment was applied. In order to avoid boundary effects at the surface, the proximal part of the electrodes was isolated leaving an active part of $15 \mathrm{~mm}$ (Fig. 1).

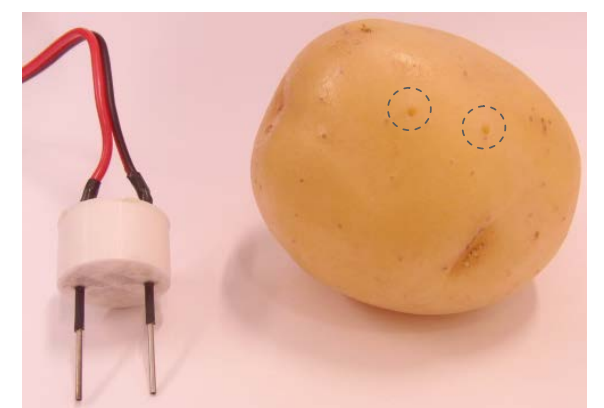

Fig. 1 Electrodes used during the experiments and a potato with holes in the peel produced by the insertion of the electrodes.

Once the IRE outcomes mainly depends on magnitude, duration and the number of pulses, it has been studied the results tuning independently each one of those parameters. It have been assessed the effect of voltage magnitude applying protocols of 10 pulses of $100 \mu$ s with voltage of 250 $(n=4), 500(n=4), 750(n=4), 1000(n=4)$ and 1250 V $(n=4)$.

On the other hand, it has been studied the outcomes with fixed voltage magnitudes $(500 \mathrm{~V})$ and duration $(100 \mu \mathrm{s})$ once $1(n=4), 3(n=4), 10(n=4), 30(n=4)$ or $100(n=4)$ pulses were delivered.

Finally in the same way, it has been studied the effect of pulse duration delivering 10 pulses of $500 \mathrm{~V}$ with durations of $10(n=4), 30(n=4), 100(n=4), 300(n=4)$ and $1000 \mu s$ $(n=4)$.

All of these electric pulse protocols were delivered at 1 $\mathrm{Hz}$ by means of a custom made generator able to deliver the specified pulse protocol.

\section{B. Sample preparation}

Potato tubers (Solanum tuberosum, Monalisa) were bought from the local supermarket. Once the electric pulses were delivered, the potato is left during 3 hours to allow the IRE cell death process. After the resting period, the whole potato was cut into slices of about $1 \mathrm{~mm}$. All the cut slices were immersed in a dye solution. It consists in a dilution of $0.5 \mathrm{~g} / \mathrm{ml}$ of commercial food dye (Wilton Ice Colorant Sky Blue) and $\mathrm{NaCl}$ solution at $0.9 \%$. The additional solute was added to obtain an isotonic solution. After 30 minutes inside the colorant solution, the slices were removed. Finally, to enhance the contrast between electroporated and nonelectroporated areas, the slices were rinsed with $\mathrm{NaCl}$ solution at $1.4 \%$. Again the solute content was used to avoid potato cells swelling.

\section{Segmentation, reconstruction and volume assessment}

With the aim of automatically analyze the slice, each one was scanned by means of a commercial desktop scanner at 600 dpi of resolution. Once digitalized, images were processed using MATLAB to obtain a binary image of both potato slice and treated area. Background was removed from the image using K-means algorithm, the whole RGB image was transformed into to $\mathrm{YCbCr}$ color space and Otsu's method [9] was used to determine the color threshold which defines the electroporated area.

The same procedure was applied for each digitalized slice obtaining a sorted stack of images (Fig. 2). Those images were oriented to avoid misalignments produced during digitalization process. After the alignment process, each slice was extruded according to the thickness. This value was obtained dividing the area of all slices by the volume of the whole tuber. The final IRE volume was calculated with the sum of the IRE areas of each slice by the thickness. 


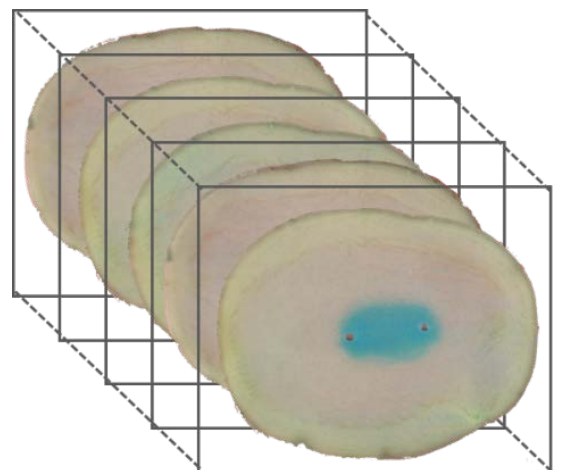

Fig. 2 Representation of an image stack composed by some of the digitalized slices obtained during the experiment.

In addition, two different tridimensional tetrahedral meshes were created from each potato, one representing the potato itself and other representing the IRE volume (Fig. $3)$. For this process iso2mesh library [10] was used.

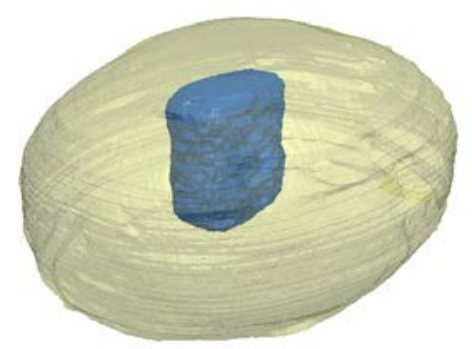

Fig. 3 Tree-dimensional images reconstruction representing the whole potato (yellow) and the IRE treated volume (blue).

\section{Numerical Simulation}

Finite element software (COMSOL Multiphysics) was used to calculate the electric field distributions within the tissue during IRE pulses. The 3D geometry used consisted on a sphere of $60 \mathrm{~mm}$ of diameter with two cylinders inserted separated $15 \mathrm{~mm}$. These cylinders have $1.27 \mathrm{~mm}$ of diameter and $15 \mathrm{~mm}$ of length. In order to represent the isolator, two additional cylinders with $2 \mathrm{~mm}$ of diameter were placed (Fig. 5).

Isolation boundary condition was established in all boundaries except on the interface between active electrodes and tissue. In this case one of the electrodes surface was defined as a ground and the other one had fixed at simulated applied voltage.

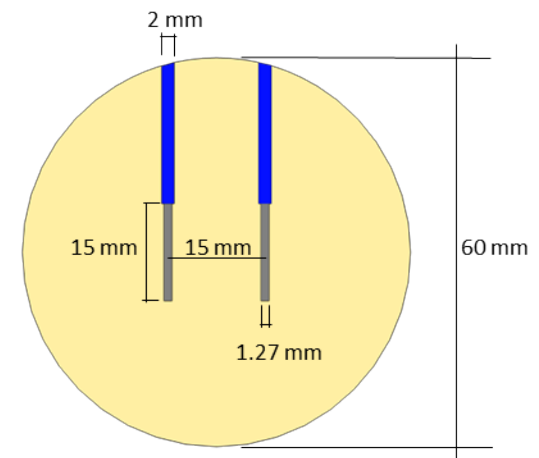

Fig. 5 Schematic representation of the geometry used for mathematical simulations where isolation (blue), active electrode (grey) and tissue (yellow) are defined.

The tissue conductivity behavior had been modeled with the following equation previously reported [5].

$$
\sigma(|E|)=0.03+0.35 \cdot e^{-e^{-0.01 \cdot(|E|-250)}}[S / m]
$$

Electric field distributions were stationary simulated with applied voltages between electrodes whose goes from 0 to $1250 \mathrm{~V}$ in steps of $50 \mathrm{~V}$. The simulated volumes of electroporated tissue were calculated according to a defined threshold value $(184 \mathrm{~V} / \mathrm{cm})$. This was obtained according the experimental results.

\section{RESULTS AND DISCUSSION}

The whole set of treated volumes obtained during the experiments can be observed in Fig. 5.

With the same number of pulses and duration, it has been observed an almost linear increase of IRE volume once the applied voltage between electrodes is raised (Fig. 5a).
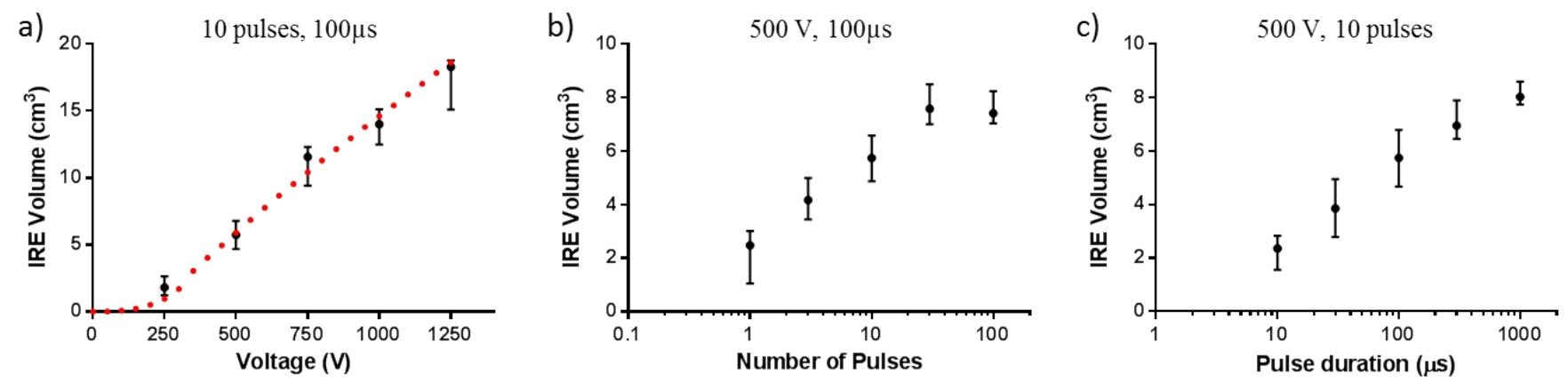

Fig. 4 Experimental (black) with median value (dot) and range value (bar). a) IRE volumes obtained during experiments for protocols with different voltage magnitude. Simulated IRE volumes (red dots) for a specified electric field threshold (184 V/cm). b) IRE volumes obtained during experiments for protocols with different numbers of pulses. c) IRE volumes obtained during experiments for protocols with different pulse duration. 
These volumes are in agreement with the values obtained in the mathematical simulations. Both experimental and simulated volumes show the same behavior regarding the voltage. This pointed out the correlation between the observed dyed areas and the real electroporated area.

On the other hand, a logarithmic behavior is observed between the assessed volumes and the number of pulses when a constant voltage and duration are applied (Fig. 5b). This result agrees to the behavior reported in other studies [6] which confirms the goodness of the experimental observations.

In the same way, the volumes obtained by incrementing the pulse duration (Fig. 5c) also show a logarithmic relation. This behavior was also observed previously [11].

Finally, it should be noted an increment of the variability in measured volumes with increasing voltage magnitudes between electrodes (Fig. 5a). This increase of variability can be attributed to the increase of total treated volume. However, in the experiments where the number of applied pulses or its duration is assessed (Fig. 5b and Fig. 5c), the variability between observations decrease despite the relative increase in the total affected volume.

\section{Conclusions}

We presented a new method for three-dimensionally asses the IRE outcomes using vegetal model. This method has been experimentally evaluated assessing the IRE treated volumes once different electric protocols were delivered. The observed values come close the theoretical predictions obtained by means of numerical simulations. It confirms the reliability of the presented evaluation method. This news assessment tool can be useful to improve both applied protocols and prediction methods, thus, contribute to improve the clinical IRE treatment outcomes.

\section{ACKNOWLEDgment}

This work received financial support from the Spanish Government through grants TEC2010-17285 and TEC201452383-C3 (TEC2014-52383-C3-2-R) and from the European Commission through the Marie Curie grant TAMIVIVE (256376).

\section{CONFLICT OF INTEREST}

The authors declare that they have no conflict of interest.

\section{REFERENCES}

1. Rubinsky B, Onik G, Mikus P (2007) Irreversible electroporation: a new ablation modality - clinical implications. Technol Cancer Res Treat 6:37-48.

2. Miklavcic D, Semrov D, Mekid H, Mir LM (2000) In vivo electroporation threshold determination. Proc 22nd Annu Int Conf IEEE Eng Med Biol Soc 4:5-8. doi: 10.1109/IEMBS.2000.901450

3. Neal RE, Millar JL, Kavnoudias H, et al. (2014) In vivo characterization and numerical simulation of prostate properties for non-thermal irreversible electroporation ablation. Prostate 74:458-468. doi: 10.1002/pros.22760

4. Arena CB, Szot CS, Garcia PA, et al. (2012) A three-dimensional in vitro tumor platform for modeling therapeutic irreversible electroporation. Biophys J 103:2033-2042. doi: 10.1016/j.bpj.2012.09.017

5. Ivorra A, Mir LM, Rubinsky B (2010) Electric field redistribution due to conductivity changes during tissue electroporation: Experiments with a simple vegetal model. IFMBE Proc. pp 59-62

6. Suárez C, Soba A, Maglietti F, et al. (2014) The Role of Additional Pulses in Electropermeabilization Protocols. PLoS One 9:e113413. doi: 10.1371/journal.pone.0113413

7. Ashie INA, Simpson BK (1996) Application of high hydrostatic pressure to control enzyme related fresh seafood texture deterioration. Food Res Int 29:569-575.

8. Pereira RN, Galindo FG, Vicente AA, Dejmek P (2009) Effects of pulsed electric field on the viscoelastic properties of potato tissue. Food Biophys 4:229-239. doi: 10.1007/s11483-009-9120-0

9. Otsu N (1979) A Threshold Selection Method from Gray-Level Histograms. IEEE Trans Syst Man Cybern 9:62-66. doi: 10.1109/TSMC.1979.4310076

10. Fang Q, Boas DA (2009) Tetrahedral mesh generation from volumetric binary and grayscale images. IEEE Int. Symp. Biomed. Imaging From Nano to Macro, ISBI 2009. pp 1142-1145

11. Pucihar G, Krmelj J, Reberšek M, et al. (2011) Equivalent pulse parameters for electroporation. IEEE Trans Biomed Eng 58:32793288. doi: 10.1109/TBME.2011.2167232

Author: Quim Castellví

Institute: Universitat Pompeu Fabra

Street: Roc Boronat 138, E-08018

City: Barcelona

Country: Spain

Email: quim.castellvi@upf.edu 